\title{
Synthesis of 1-Substituted-phytosphingosine : Novel Protection of Phytosphingosine
}

\author{
Su Yeon Jo, Hỵoung Cheul Kim, Seung Woo Woo, Min Jung Seo, Gehyeong Lee, and Hyoung Rae Kim \\ Medicinal Science Division, Komea Research Instinte of Chemical Technologe, P.O. Bow 107 , \\ Husong. Dajeon 305-600. Korea \\ Received December 15,2002
}

Key Words : l'hytosphingosine, l-Substituted-phytosphingosine, l'lyytosphingosine l-phosphate, Sphingosine, Cyclic carbonate

Phytosphingosine. one of the major sphingosine derivaives was found in microorganisms. ycasi. plants. and fungi as a major membrane component. and also found in many mammalian tissues ${ }^{3}$ and interestingly in some cancer cell1ypes. ${ }^{2}$ The roles of sphingosine derivatives in human cells have been enigmatic but they are recently proved to be essential in cell communications and regulation of cell growth. ${ }^{3}$ Since the sphingosine derivatives are very interesting both biologically and synthetically, many attempts have been tried to synthesize splingosine derivalives. ${ }^{-1}$ howerer cxamples of modifications of sphingosine derivatiyes are very rare ${ }^{56}$

In plỵtosphingosine (1). there exist two major parts. long lincar by drocarbon chain and very hydrophilic terminal with an amine and three ly droxyl groups. In order 10 modify the structure of phytosphingosine. proper protections for the amino and hydroxyl groups are essential. In this paper. we describe a novel protection of plytosplingosine and some example syntheses of new plyylosplingosine derivatives.

Since plyy tosphingosine (1) itsclf has very low solubility in various organic solyents and water. first protection of amino group by di-t-buly] dicarbonatc was only successfully perforned in t-butyl alcohol. The resulting X-Boc-plyytosplingosine (2) was casily separated as wlite precipitates in $94 \%$ yicld by the craporation of solyent and only washing will n-

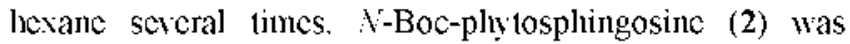
reacied will phenyl chlorofonnate and pyridine at $0^{\circ} \mathrm{C}$ in THF 10 afford plicnyl carbonate 3 in good yicld. To the reaction mixture was added 1.2 cquiv of DBU at $25^{\circ} \mathrm{C}$. the carbonate at 1-position was migrated to 3-position. and then the $3.4-c y c l i c$ carbonate 4 was fonned as a white solid in tolal $67 \%$ yicld after purification by silica gel column chromatography (mellỵlenc chloride/methanol. 40/1) as shown in Scheme 1. Though cxecss phenyl clilorofonnate was used in pyridinc. only 3 was obtained without any formation of byproducls. Plicnyl carbonate 3 was stable in py ridine and it could not be comerted to cyelic carbonate 4 wilhoul DBU at $25^{\circ} \mathrm{C}$.

We cxamined other bases to oblain 1.3-cyclic carbonate from 3. bul only 4 was oblained withoul any trace of 1.3cyclic carbomatc. Hydrolysis of + with potassium carbonatc in $95 \%$ mellanol quantitatively afforded $\lambda$-Boc-phytosplingosine (2) and from these results the siercochemistry of 4 was confinned. The spectroscopic data of all compounds were summarized in the references. The compound is propery protected plyytosphingosine derivative. which can
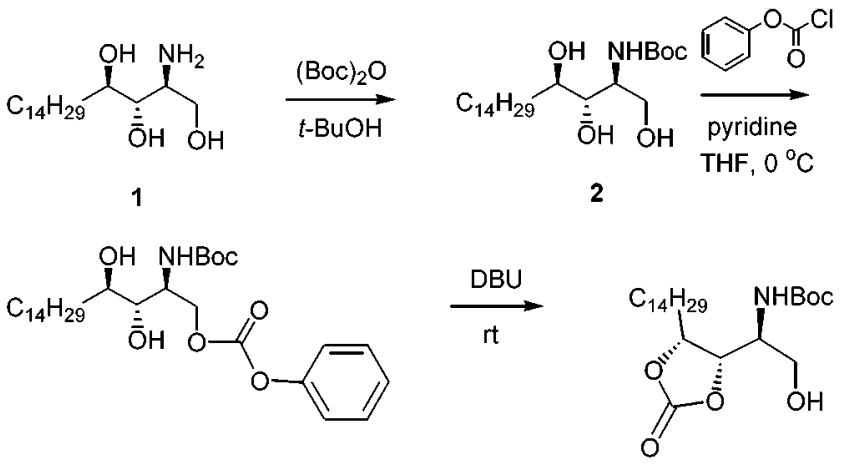

3

4

Scheme 1

be used as a starting matcrial for the derivatizations of phỵlosplingosine at 1-position.

To cxplore the substitutions at 1-position of plytosphingosinc. [irst we tricd to prepare plyy tosphingosinc-1-phosphatc (7) as shown in Scheme 2. The cyclic carbonate 4 was reacted with trimethy] plosphite and carbon tetrabromide at $0{ }^{\circ} \mathrm{C}$ in py ridine to afford the phosphate $5 \mathrm{in} 60 \%$ yicld. and the removal of carbonate group of 5 were casily performed by potassium carbonate in $95 \%$ methanol at $40^{\circ} \mathrm{C}$ within $1 \mathrm{~h}$ 10 give 6 as a pale ycllow oil in $94 \%$ yicld. $X$-Boc group and mellyy] csicr of 6 were removed simultancously by 5 cquiv of bromolrimetlyylsilanc ${ }^{5 \text { tb }}$ followed by addition of small water to afford 7 as a white precipitate in $40 \%$ yicld. The speciral data of 7 showed good coincidence with those in the references. 6

Next we mesylated the cyclic carbonate t. and the resulting 8 was reacled with nuclcophiles such as sodium

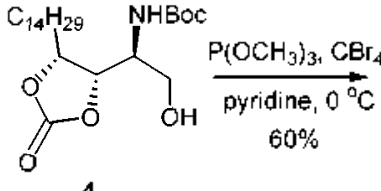

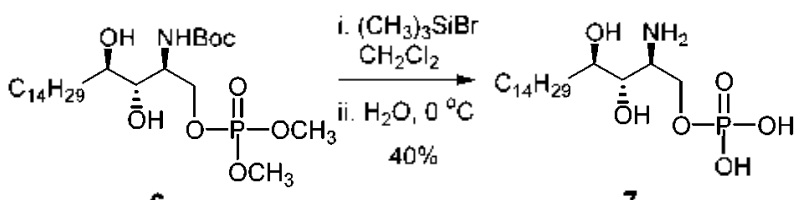<smiles>COP(=O)(OC)OCC(NC(=O)OCc1ccccc1)[C@H]1OC(=O)O[C@@H]1C</smiles>

5
Scheme 2 
<smiles>C[C@@H]1OC(=O)O[C@@H]1[C@H](CO)NC(=O)OC(C)(C)C</smiles>

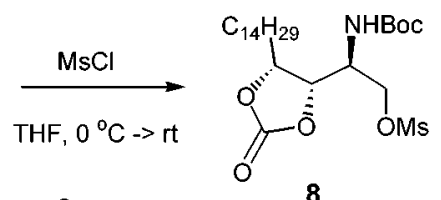

8

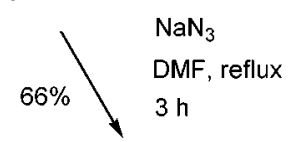<smiles>CCCCCCCCCCC(=O)NCCN</smiles>

10
Scheme 3

azide and cesium thioacetate (Scheme 3 ). 8 was reacted with 1.2 equix of cesium thioacetate in DMF at $25^{\circ} \mathrm{C}$ for 2 days. and addition of small amount of water to the reaction mixture gave thiol 9 as white precipitates in 94\% yield. which could be identified by upfield shift of $\mathrm{C}-1$ protons (3.01 and $3.29 \mathrm{ppm}$ ) compared with $+(3.73$ and $3.91 \mathrm{ppm})$ in ${ }^{1} \mathrm{H}$ NMR. Similarly. 8 was reacted with 1.2 equiv of sodium azide in DMF with heating for $3 \mathrm{~h}$. and addition of small amount of water to the reaction mixture gave azide $\mathbf{1 0}$ as pale yellow precipitates in $66 \%$ yield, which also could be identified by upfield shift of $\mathrm{C}-\mathrm{l}$ protons $(3.42$ and 3.50 ppm) compared with 4 (3.73 and 3.91 ppm) in ${ }^{1} H$ NMR. lnterestingly. when electron-withdrawing groups such as esters. carbonates, and sulfonates were substituted at C-l. $\mathrm{C}-2$. $\mathrm{C}-3$. or $\mathrm{C}-4$ in plyytosplingosine, protons at those positions appeared over + ppm. while unsubstituted hydroxyl groups and amine in plytosplingosine appeared below + ppm in 'H NMR spectrum.

In summary, plytospluingosine was protected by the formation of cyclic carbonate in two steps. which could be useful for the derivatizations of 1-position of phytosphingosine. Phytosphingosine-l-phosphate and other derivatives of plyytosphingosine were synthesized from the phytosphingosine derivatives protected with cyclic carbonates.

\section{References and Notes}

1. (a) Karlsson. K. A. feta (Them. Seand 1964. 18. 2295. (b) Karlsson. K. A. Acto Chem. Scomd. 1964, 19, 2297. (c) Karlsson, K. A.; Samulsson, B. F.: Stecn, G. O. Acta Chem. Scomd. 1968, 22. 1361. (d) Wert7. P. W: Mictlike, M. C.: I ong. S. A.: Stauss, J. S.: Downing. D. T. I. Inest. Dermatol 1985. \&t. 410 . (e) Sclmidt. R. l'. In Fiposome Deymatics: Braun-Falco. O. H.: Corting. C.: Maibach. H. 1.. Eds.: Springer-Verlag: Berlin. Heidelbere. 1992: pp 44-56. (f) Batenhol7. Y: Gatt. S. Biochem. Biophns. Res. Commm. 1967, 27,319. (g) Takamatsu, K.: Mikami, M.: Kikuchi, K.: Norawa, S.: Iwamori, M. Biochm. Biophes. Acta 1992, 1165 . 177. (h) Okabe. K.: Keenan. R. W.: Schmidt. G. Biochem. Biophys. Res Commm 1968. 3\% 137. (i) Vance. D. E.: Sweeley. C. C. J LipidRe?s. 1967. B.621.

2. Kanfer. J. N.: IJakomon, S. In Handbook of Lipid Researh tot. Sphingolipid Biochemismy: IJakomori, S., Fd.; Plenum Press: Now York, 198.3.

3. (a) Lee. Y. J.: Hannun. Y. A.: Obeid. L. M. I. Biol. Chtem. 1996.
271. 13169. (b) Hannun. Y. A. J. Biol (hem. 1994. 269.3125. (c) Gomez-Muncz. A.: Waggoner. D. W.: O'Brien. L.: Brindley. D. N. J. Biol (hem. 1995. 270. 26318. (d) Merill dr.. A. H. Kitr: Re?.

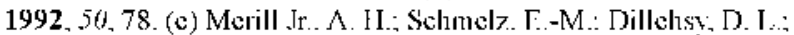
Spiegel. S.: Shayman, J. A.: Schroeder, J. J.: Riler: R. T.: Voss, K. $\Lambda$.: Wang. F. foxicel. Appl Tharmacol 1997, 1f2, 208. (t) Merill Ir. A. H. J. Bioenerg. Bionembr, 1991. 23.83.

4. (a) Korkinen. P. M.: Koskinen. A. M. P?. Synthesis 1998. 1075, (b) He. L.: Byun. H.-S.: Bitleman. R. J. Org. (hem. 2000. 65. 7618. (c) Chun, I.: IJe, I..; Bsun, II.S.: Bitterman, R. J. Org. Chem. 2000, 65. 763.34

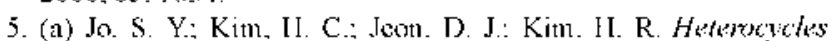
2001. 55. 1127. (b) Szule. L. M.: Hannun. Y. A.: Bielawskit. A. Tetrathedron lett. 2000. H. 7821 .

6. (a) Boumendjel. A.: Miller. S. P. F. J. Lipid Res. 1994. 35. 2305. (b) I.i. S.: Wilson, W. K.: Schrocpter, Ir. G. I. J. Lipid Res. 1999. 40. 117 .

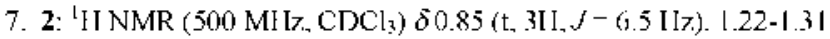
(m. $24 \mathrm{H}) .1 .42(\mathrm{~s} .9 \mathrm{H}) \cdot 1.50-1.58(\mathrm{~m} . \mathrm{HH}) .1 .60-1.71(\mathrm{~m} .1 \mathrm{H}) .3 .61-$ 3.67 (m. 3H). $3.72-3.76$ (m. $2 \mathrm{H}) .3 .86$ (dd. $2 \mathrm{H} . J-11.2 .2 .8 \mathrm{~Hz})$. 4.17 (br. $1 \mathrm{H}$ ). 5.24 (d. IH. $J-8.3 \mathrm{H} \angle . \mathrm{NH}$ ): IR (NaCl) 3326.4 . 2916.9. 2847.9. 1167.3. 1545.2. 1481.9. 1356.6. 1252.2. 1171.1. $1057.9 \mathrm{~cm}$ ': MS m: 7 (CI. relatice intensity) 418 (M'. 39), $390(6)$.

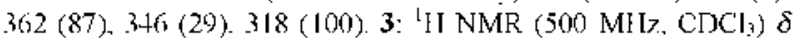
$0.85(\mathrm{l} .3 \mathrm{H} . J-6.9 \mathrm{H} \angle) .1 .22-1.31(\mathrm{~m} .24 \mathrm{H}) .1 .42(\mathrm{s.} 9 \mathrm{H}) .1 .46-1.52$ (m. lH). $1.58-1.70$ (m. IH). 2.57 (br. IH). 3.12 (br. IH). 3.61-3.64 (m. 2H). $4.06-4.07$ (m. 1H). 4.46 (dd. $2 \mathrm{H} . J-10.0 .3 .2 \mathrm{~Hz}$ ). 5.23 (d. III $J-9.1$ IIz. NII). $7.14-7.15$ (m. 2II). $7.20-7.24$ (m, IH I). 7.3.3$7.36(\mathrm{~m}, 2 \mathrm{II}): \mathrm{IR}(\mathrm{NaCl}) 2924.1,2853.0,1765.5,1689.2 .1366 .2$. 1249.2. $1166.3,1047.2 \mathrm{~cm}$ ': MS m: . (CI, relatife intensity) 416 (M -l'hoC-0.9). $388(89) .370(5) .344$ (9). $326(12) .95$ (100). 4: ${ }^{1} \mathrm{H}$ NMR $\left.(500 \mathrm{MHz} . \mathrm{CLCl})_{3}\right) \delta 0.85(\mathrm{l} .3 \mathrm{H} . J-6.5 \mathrm{~Hz}) .1 .22-1.31$ (m. $24 \mathrm{H}$ ). 1.42 (s. 9H). $1.56-1.72$ (m. $2 \mathrm{H}) .2 .72$ (br. $1 \mathrm{H}) .3 .73$ (d. III. $J-4.9$ IJ $) .3 .91$ (d. III $J-4.9$ IJ). $3.96-3.99$ (m. III) $4.68-$ $4.7 \mathrm{I}$ (m. 2I I). 4.97 (br. 1H): ${ }^{13} \mathrm{C}$ NMR (125 MI I7, $\left.\mathrm{CDCl}_{3}\right) \delta 14.08$. $22.65 .23 .15,25.50,28.53 .29 .10,29.32,29.39 .29 .49,29.60 .29 .62$. 29.65. 29.66. 31.88. 48.55.61.53. 77.52.80.32. 154.70. 170.53: IR (NaCl) 3298.9. 2918.5. 1806.1. 1657.5. 1562.0. 1462.2. 1372.0 . $1058.1 \mathrm{~cm}^{-1}$ : MS mะ (Cl. relative intensity) $+16\left(\mathrm{M}^{+}-\mathrm{C}-0.23\right)$. $388(100) .370(77) .344$ (17). $326(25) .95$ (62): Anal. Calcd tor $\mathrm{C}_{21} \mathrm{II}_{15} \mathrm{NO}_{11}: \mathrm{C}, 64.98: \mathrm{HI}, 10.22 ; \mathrm{N}$. 3.16. Found: C, 65.01: H, 10.32; N. 3.08. 5: ${ }^{\mathrm{H}} \mathrm{I}$ NMR (500 MI I7., $\left.\mathrm{CDCl}_{3}\right) \delta 0.87($ t. $3 \mathrm{II}, J-6.9 \mathrm{lJ})$ ), $1.18-1.29$ (m. $24 \mathrm{H}) .1 .43$ (s. $9 \mathrm{H}) .1 .50-1.57$ (m. $2 \mathrm{H}) .3 .79$ (d. $3 \mathrm{H} . J$ $-2.6 \mathrm{~Hz}$ ). 3.82 (d. $3 \mathrm{H} . J-2.6 \mathrm{~Hz}$ ). $4.27-4.29$ (m. IH). $4.26-4.30$ (m. IH). $4.68-4.75$ (m. 2H). 5.24 (d. $1 \mathrm{H} . J-9.5 \mathrm{H} z$. NH): MS m $\angle$ (relative intensity) $554\left(\mathrm{M}^{-}, 11\right), 5.39(2+), 52+(98) .480(.77)+.36$ (8). $370(14) .326(16) .155(100)$. 6: 'II NMR (500 Ml I7. CDCl $\mathrm{CD}_{3} \delta$

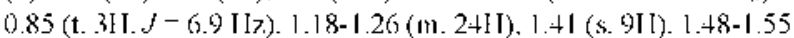
(m. $2 \mathrm{H}) .3 .40$ (br. $2 \mathrm{H}) .3 .55-3.68(\mathrm{~m} .2 \mathrm{H}) .3 .72$ (s. $3 \mathrm{H}) .3 .78(\mathrm{s.} 3 \mathrm{H})$. $3.81-3.98(\mathrm{~m} . \mathrm{lH}) .4 .16-4.21(\mathrm{~m} . \mathrm{HH}) \cdot 4.23-4.40(\mathrm{~m} .1 \mathrm{H}) .5 .42(\mathrm{~d}$. IH. $J-4.5 \mathrm{~Hz} . \mathrm{NH}), 8$ : 'H NMR ( $\left.500 \mathrm{MHz} . \mathrm{CDCl}_{3}\right) \delta 0.85$ (c. 3H. $J-6.9$ IJ), 1.22-1.28 (m. 24II). 1.40 (s. 9H). 1.57-1.7l (m. 2H), $3.05(\mathrm{~s}, 3 \mathrm{II}) .4 .16-4.23(\mathrm{~m}, 1 \mathrm{H}), 4.36$ (dd. $2 \mathrm{I}, J-5.3$. $10.4 \mathrm{I}(\mathrm{z})$ ). $4.64(\mathrm{t}, 1 \mathrm{II}, J-10.0 \mathrm{II}$ ), 4.71 (dd, III,J-3.4.9.2 I J ). 4.96 (d, III, J $-5.3 \mathrm{~Hz} . \mathrm{NH})$ : IR (NaCl) 3359.4. 2925.5. 2854. 1. 2360.5. 2341.2. 1810.8. 1717.9. 1523.5. 1365.4. 1174.4. $1050.2 \mathrm{~cm}^{-1}, 9:{ }^{1} \mathrm{H}$ NMR $\left(500 \mathrm{MHz} . \mathrm{CDCl}_{3}\right) \delta 0.88$ (L. $\left.3 \mathrm{H} .7-6.9 \mathrm{~Hz}\right) .1 .23-1.29(\mathrm{~m} .24 \mathrm{H})$. 1.4 .3 (s. 9I), 1.69-1.82 (m, 2H, 3.01 (dd. III, $J-9.5$. 14.2 I lz). 3.29 (dd, III, $J-2.9,14.2 \mathrm{I}$ (7.). $4.02-4.10$ (br, III, NI I), $4.57-4.65$ (111, 2I I), 4.69 (dd, III, $J-7.3,13.8 \mathrm{I}$ (7): IR (NaCl) 2920.3. 2870.0 . 2359.0. 2339.9. 1808.1. 1696.4. $763.0 \mathrm{~cm}$ '. 10: 'H NMR (500 $\mathrm{MHz} . \mathrm{CDCl}_{3}$ ) $\delta 0.85$ (t. $3 \mathrm{H} . J-7.1 \mathrm{~Hz}$ ). $1.23-1.32$ (m. $24 \mathrm{H}$ ). $1.50-$ $1.65(\mathrm{~m} .2 \mathrm{H}) .1 .57(\mathrm{~s} .9 \mathrm{H}) .3 .42(\mathrm{dd} .1 \mathrm{H} .7-6.4 .12 .4 \mathrm{~Hz}) .3 .50(\mathrm{dd}$. III. $J-5.0,12.4 \mathrm{H}$ ), $3.52-3.57$ (m, IH), .3.89-3.94 (m. III), 4.18 (dd, III, $J-3.3,5.3 \mathrm{HI}$ ). 5.47 (br, III, NII): IR (NaCl) 2922.1,

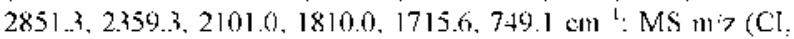
relative intensity) $4+1\left(\mathrm{M}^{-}-\mathrm{C}-\mathrm{O} .11\right)+413\left(\mathrm{M}^{\prime}-\mathrm{C}-\mathrm{O}-\mathrm{N}_{2} .100\right) .370$ (5). $370(9) .338(8) .312(35)$. 